\title{
A multi-site, randomized study of strengths-based case management with substance-abusing parolees
}

\author{
Michael Prendergast • Linda Frisman • \\ JoAnn Y. Sacks • Michele Staton-Tindall • \\ Lisa Greenwell • Hsiu-Ju Lin • Jerry Cartier
}

Published online: 21 April 2011

(C) The Author(s) 2011. This article is published with open access at Springerlink.com

\begin{abstract}
Objectives To test whether strengths-based case management provided during an inmate's transition from incarceration to the community increases participation in community substance abuse treatment, enhances access to needed social services, and improves drug use, crime, and HIV risk outcomes.

Methods In a multi-site trial, inmates (men and women) in four states $(n=812)$ were randomly assigned (within site) to receive either Transitional Case Management (TCM group), based on strengths-based principles, or standard parole services (SR group). Data were collected at baseline and at 3 and 9 months following release from prison. Analyses compared the two groups with respect to services received and to drug use, crime, and HIV risk behavior outcomes.

Results There were no significant differences between parolees in the TCM group and the SR group on outcomes related to participation in drug abuse treatment, receipt of social services, or drug use, crime, and HIV risk behaviors. For specific services (e.g., residential treatment, mental health), although significant differences
\end{abstract}

M. Prendergast $(\bowtie) \cdot$ L. Greenwell $\cdot$ J. Cartier

Integrated Substance Abuse Programs, University of California Los Angeles, 1640 S. Sepulveda Blvd., Suite 320, Los Angeles, CA 90025, USA

e-mail: mlp@ucla.edu

L. Frisman $\cdot$ H.-J. Lin

Research Division, Connecticut Department of Mental Health \& Addiction Services,

Hartford, CT, USA

J. Y. Sacks

Center for the Integration of Research \& Practice, National Development \& Research Institutes, Inc, New York, NY, USA

M. Staton-Tindall

Center on Drug and Alcohol Research, University of Kentucky, Lexington, KY, USA

Present Address:

L. Greenwell

RMC Research Corporation, Portland, OR, USA 
were found for length of participation or for number of visits, the number of participants in these services was small and the direction of effect was not consistent. Conclusion In contrast to positive findings in earlier studies of strengths-based case management with mental-health and drug-abuse clients, this study found that case management did not improve treatment participation or behavioral outcomes for parolees with drug problems. The discussion includes possible reasons for the findings and suggestions for modifications to the intervention that could be addressed in future research.

Keywords Case management · Drug-use offenders · Experimental design · Field experiments $\cdot$ Offender treatment

\section{Introduction}

A growing body of research indicates that prison-based substance abuse treatment needs to be followed by community treatment in order to achieve optimal outcomes (Hall et al. 2003; Knight et al. 1999; Martin et al. 1999, 2003; Wexler et al. 1999). As a result, the correctional treatment system of many states consists of a continuum of care, in which inmates with substance abuse problems participate in prison-based treatment, followed by treatment in a community-based program. To link these two phases of treatment, a planning and transition process involving correctional or treatment staff and the inmate usually occurs prior to release to the community. Depending on the correctional system, referral to community-based treatment consists of either encouraging inmates with drug problems to volunteer for community treatment or mandating them to treatment as a condition of parole. Too often, however, the transition process breaks down from individual and systemic reasons.

Low participation rates by parolees in community treatment are a barrier to the overall effectiveness and cost effectiveness of offender treatment programs. Improvements in offender behavior from continued treatment in the community can be realized only if prison treatment participants follow through on referrals to community treatment and remain in treatment for a minimum length of time, generally considered to be at least three months (Hubbard et al. 1989; Simpson et al. 1997). Failure of parolees to enter or remain in treatment often results in a greater likelihood of relapse to drug use or reincarceration. Adherence to treatment requirements by parolees, particularly when treatment is voluntary, is often lax for a variety of reasons: high parole caseloads, lack of priority by parole agents for treatment, poor coordination between criminal justice and community treatment personnel, and low motivation and accountability on the part of parolees. Thus, there is a need to increase the likelihood that inmates with a referral to community treatment enter their assigned treatment program and remain engaged in treatment for a reasonable length of time (e.g., 90 days). Increased participation should, in turn, be associated with improved longer-term outcomes in drug use, crime, and other behaviors.

Successful transition from prison to community treatment is likely to result from several processes, including (1) enhancing the engagement and motivation of the prisoner during planning for community treatment, (2) fostering collaboration between prison, parole, and treatment staff during the parolee's re-entry to the 
community, (3) ensuring continuity of care between treatment services obtained in prison and in the community, and (4) providing initial post-release support to the client to facilitate admission to treatment (Hammett et al. 2001; Prendergast 2009; Taxman 2004). These processes can be addressed at two levels: (1) improving coordination and collaboration among staff in prison, parole, and treatment and among health, mental health, and social service providers and (1) working directly with the offender to develop specific goals and plans for re-entry and by assisting him/her during the crucial early months on parole.

Of various options that might be used to achieve these ends, case management is a promising approach. Active case management strategies, in which the case manager begins working with the inmate in the institution and continues to do so in the community, may be an effective way to overcome both system and individual obstacles in the transition and re-entry process and to foster improved post-release adjustment. As discussed further below, the study reported on here, implemented in four states, used a randomized design to compare a strengths-based case management model (Hall et al. 1999; Siegal and Rapp 1996) to improve the reentry process for substance-abusing offenders with standard parole supervision. The Transitional Case Management (TCM) study was one of the multi-site studies conducted as part of the Criminal Justice Drug Abuse Treatment Studies (CJ-DATS) cooperative, funded by the National Institute on Drug Abuse.

The discussion below begins with a description of strengths case management, why it might be appropriate for a parolee population, and what previous studies have found with respect to the effectiveness of case management. The two study conditions are then described, along with a summary of the training and monitoring of the case managers. There follows a detailed description of the design of the study and the methods of analysis. The "Results" section provides descriptive data on the intervention and results from tests of the study hypotheses. Finally, the "Discussion" highlights key issues in the design and findings that bear on interpreting the results and understanding the broader significance of the study.

\section{Strengths-based case management}

A variety of case management models exist (e.g., brokerage, assertive case management, clinical case management, intensive case management), but the model that is the focus of this study is "strengths-based case management" (Hall et al. 1999; Saleebey 2002; Siegal et al. 1995). Although all case management models have common elements (e.g., assessment, planning, referral, advocacy, monitoring; Ridgely et al. 1996), strengths-based case management differs from other models in a number of ways, as indicated in Table 1 (adapted from Ridgely and Willenbring 1992 and Hall et al. 2002). Like most case management models, the strengths-based model was originally developed for persons with mental illness, but it has subsequently been adapted for other populations, including clients in substance abuse treatment.

Six principles characterize strengths-based case management, as formulated by Rapp and Wintersteen (1989):

1. The focus is on the strengths of the client, not on pathology or deficits. 
Table 1 Characteristics of strengths case management in the TCM study in comparison with lowand high-intensity case management models

\begin{tabular}{|c|c|c|c|}
\hline Characteristics & $\begin{array}{l}\text { Low-intensity case } \\
\text { management }\end{array}$ & $\begin{array}{l}\text { Strengths case } \\
\text { management }\end{array}$ & $\begin{array}{l}\text { High-intensity case } \\
\text { management }\end{array}$ \\
\hline Duration & Time limited & $\begin{array}{l}2 \text { months in prison; } 3 \text { months in } \\
\text { community weekly; } 3 \text { months in } \\
\text { community monthly }\end{array}$ & Indefinite \\
\hline $\begin{array}{l}\text { Frequency } \\
\text { of contact }\end{array}$ & $\begin{array}{l}\text { Infrequent (quarterly } \\
\text { contact) }\end{array}$ & $\begin{array}{l}2 \text { contacts in prison; weekly } \\
\text { for } 3 \text { months, then monthly } \\
\text { for } 3 \text { months }\end{array}$ & Frequent (daily contact) \\
\hline $\begin{array}{l}\text { Staff:Client } \\
\text { ratio }\end{array}$ & High $(1: 75)$ & Medium (1:15-20) & Low $(1: 10)$ \\
\hline $\begin{array}{l}\text { Focus of } \\
\text { services }\end{array}$ & Narrow; exclusive & $\begin{array}{l}\text { Broad: treatment participation, client } \\
\text { goals and needs as determined } \\
\text { by Strengths Assessment } \\
\text { and Goal Plan }\end{array}$ & Broad: inclusive \\
\hline $\begin{array}{l}\text { Type of } \\
\text { service }\end{array}$ & $\begin{array}{l}\text { Management of services } \\
\text { provided by others }\end{array}$ & $\begin{array}{l}\text { Manage and coordinate services provided } \\
\text { by other agencies }\end{array}$ & Provides all services \\
\hline Availability & Office hours & $\begin{array}{l}\text { Mainly office hours, some } \\
\text { evenings and weekends }\end{array}$ & 24 hours \\
\hline $\begin{array}{l}\text { Site of case } \\
\quad \text { management } \\
\text { services }\end{array}$ & Office only & $\begin{array}{l}\text { In office, in the community, } \\
\text { and by telephone }\end{array}$ & In community \\
\hline $\begin{array}{l}\text { Client } \\
\text { direction }\end{array}$ & Professionally directed & $\begin{array}{l}\text { Largely client directed, subject to parole } \\
\text { and treatment requirements }\end{array}$ & Client directed \\
\hline Advocacy & $\begin{array}{l}\text { Gatekeeper for system } \\
\text { (finds alternatives to } \\
\text { requested services) }\end{array}$ & $\begin{array}{l}\text { Case manager advocates for client } \\
\text { to obtain access to services }\end{array}$ & $\begin{array}{l}\text { Advocates for client } \\
\text { (to gain access to services) }\end{array}$ \\
\hline Training & On-the-job training & $\begin{array}{l}\text { Project-provided training, with } \\
\text { ongoing supervisory support }\end{array}$ & Advanced professional degree \\
\hline Authority & $\begin{array}{l}\text { No authority, persuasion } \\
\text { only }\end{array}$ & No authority, use of persuasion only & $\begin{array}{l}\text { Broad authority, } \\
\text { administrative control }\end{array}$ \\
\hline Team structure & $\begin{array}{l}\text { Primary case manager } \\
\text { with individual } \\
\text { caseload }\end{array}$ & $\begin{array}{l}\text { Case manager with individual } \\
\text { case load }\end{array}$ & $\begin{array}{l}\text { Full team mode: all case } \\
\text { managers share all clients. }\end{array}$ \\
\hline
\end{tabular}

2. The relationship between the case manager and the client is an essential component.

3. Interventions and services are determined by the needs and desires of the client.

4. The preferred mode of intervention for the case manager is aggressive outreach.

5. All people, regardless of their current condition or situation, are able to learn, grow, and change, and the role of the case manager is able to assist in this process.

6. The entire community (including formal and informal resources) is viewed as a source of services and support for clients.

Drawing on these principles, the strengths approach involves establishing a relationship between the client and the case manager that is different from other models. The quintessential component of strengths case management is the identification of the client's strengths and previous accomplishments, rather than focusing on deficits, problems, or obstacles, and the repeated utilization of these strengths during each session as a way of achieving goals and addressing unexpected barriers. In contrast to a deficit model that reinforces low expectations by viewing 
clients as 'the problem' (e.g., one is a drug addict), the strengths approach reinforces high expectations by viewing the situation as 'the problem' (e.g., one has drug dependence) and by assuming that the client has many strengths and resources with which to handle the problem (Rapp 1992). The case manager and client collaborate to assist the client in setting and achieving goals that the client (rather than the case manager or some other person or agency) identifies as valuable and important (Kisthardt 2002). Thus, the case manager works with the client to improve selfsufficiency within a supportive environment and serves as a 'bridge' between the client and an often fragmented and difficult-to-access service system. In addition, the strengths model looks beyond formal (usually government-supported) services to the rich array of informal resources available in the community such as families, faithbased organizations, volunteer organizations, and self-help groups (Saleebey 1996). Like other case management models, strengths case management is a "boundary spanning" intervention. Boundary spanning is a concept in organizational and management research that refers to a task or series of tasks that requires communication and interaction among people within different agencies or systems who, because of contrasting goals, training, or skills, "speak different languages" (Kerson 2001). As such, the case manager helps to coordinate services for the client (in this study, a parolee) among multiple agencies.

For a parolee population, the strengths-based approach has a number of advantages over other case management models. First, as opposed to less intensive forms of case management that rely mainly on referral to services, the case manager in the strengths-based model actively assists and advocates for the client during the crucial first months of parole. Second, strengths case management fosters selfmanagement skills so that clients may eventually become their own 'case manager,' capable of exercising greater self-sufficiency in seeking services and resources once case management assistance has ended. Third, strengths case management is less dependent on a team approach compared with more intensive (and expensive) forms of case management. Finally, the focus of strengths case management on assets, accomplishments, and goal setting seems particularly appropriate for parolees who have come out of prison-with its climate of coercion, stigmatization, and dependency - and who are attempting to adjust successfully to community living.

Case management interventions (not specifically strengths based) have been found to be effective in linking with substance-abusing populations with treatment (Coviello et al. 2006; Rapp et al. 2008; Sorensen et al. 2005) and in addressing substance abuse problems (Siegal et al. 1996), employment (Martin and Inciardi 1993; Siegal et al. 1996), and criminality (Siegal et al. 2002). With specific reference to the objectives of this study, Mejta et al. (1997) found, in a randomized study, that intravenous drug users in Chicago who participated in case management entered treatment in higher numbers, entered treatment more rapidly, and remained in treatment longer than did those in the standard referral condition.

Case management using strengths-based principles has been examined in several NIDA-funded studies. In one study (Siegal et al. 1996, 2002), substance abuse treatment clients were randomly assigned to primary care plus standard aftercare services or to primary care plus aftercare enhanced with strengths case management. The strengths-based approach to case management was effective in increasing retention in treatment, which in turn had a significant positive impact on post- 
treatment drug use, criminality, and employment. In another randomized study, Vaughan-Sarrazin et al. (2000) found that substance abuse clients with strengthsbased case managers reported receiving more treatment aftercare services and medical services compared with clients who received standard treatment (see also Hall et al. 1999). Strathdee et al. (2006) compared the effectiveness of strengthsbased case management and passive referral in increasing treatment participation among injecting drug users referred from a needle exchange program. Although less than half of all subjects entered treatment within 7 days of referral $(35 \%)$, a significantly higher percentage of case management clients than passive referral clients entered treatment within this period (40 vs. $26 \%$ ).

A recent meta-analysis quantitatively combined the results from 15 randomized studies of case management for persons with substance use disorders (Hesse et al. 2007). For studies in which case management (all types of models) was compared with treatment as usual (eight comparisons), the authors found a moderate effect size for the ability of case management to link clients with treatment and other services $(d=0.42,95 \% \mathrm{CI}=0.21,0.62)$. For more distal outcomes measured at follow-up, however, the effects tended to be small and not significant (possibly due to the relatively small number of studies). The effect size $(d)$ for illicit drug use outcomes (eight comparisons) was $0.12(95 \% \mathrm{CI}=-0.06,0.29)$ and for legal status (four studies), $0.05(95 \% \mathrm{CI}=-0.05,0.15)$. The meta-analysis did find that the effect on linkage with services was improved in studies that used manuals. Studies of strengths-based case management specifically had a large effect size for linkage $(d=0.70,95 \% \mathrm{CI}=0.31,1.08)$, but a small effect size for illicit drug use $(d=0.24$, $95 \% \mathrm{CI}=0.06,0.42$ ). Both effect sizes were significant, although based on only two studies for linkage and one study for drug use. (Eligibility criteria for inclusion in the meta-analysis eliminated some empirical studies of strengths case management; e.g., Vaughan-Sarrazin et al. 2000.)

In summary, the research literature on case management for substance abusers indicates that this approach is most effective in forging linkages with services and that it also has an impact, though more modest, on longer-term drug use and crime outcomes. The philosophy and activities of the strengths model of case management seems particularly appropriate to assist parolees in dealing with their substance use and other needs during the early months of re-entry. This study was intended to determine whether strengths case management is able to improve parolees' participation in community treatment and their access to other services and thereby impact longer-term outcomes, compared with parolees under standard parole supervision. To our knowledge, this is the first study to assess the effectiveness of strengths case management with an exclusively parolee sample, as opposed to a general substance abuse sample that includes offenders (usually probationers).

\section{Overview of the study conditions}

\section{Standard referral group}

Inmates who were assigned to the Standard Referral group received the usual planning and referral services available in prison and while on parole, including (in 
all study sites) a referral to community-based treatment. As inmates prepared to leave prison, institutional or treatment staff facilitated access to community-based treatment by identifying service agencies in the offender's planned living community and making a referral to community agencies as a means of providing a linkage between the institution and community. Once in the community, the participants received standard supervision and services through their parole officer.

\section{Transitional case management group}

In addition to receiving the usual referral and supervision services listed above, inmates assigned to the TCM group received the services specified in the TCM protocol (see Prendergast and Cartier 2008 for more details on the protocol). The TCM intervention consisted of three components intended to promote treatment participation and facilitate access to services. First, the intervention included processes and activities that are standard in case management models: assessment, planning, referral, linkage and coordination, advocacy, and monitoring (Ridgely et al. 1996). Second, the TCM intervention was based on the philosophy of strengthsbased service delivery as embodied in the six principles listed above. Third, the interaction between the client and the case manager was operationalized in terms of solution-focused therapy (de Shazer 1988; Gingerich and Eisengart 2000; Miller et al. 1996), which is a structured behavioral approach that is philosophically and therapeutically compatible with strengths case management. Solution-focused therapy builds on client strengths, emphasizes solutions rather than problems, breaks goals into specific activities, and identifies the person(s) responsible for each activity.

The TCM intervention included three phases, the first two of which occurred in the institution and the third in the community:

- Strengths Assessment. At the first session, conducted about two months prior to release, the case manager met with the client to complete a Strengths Assessment inventory that identified strengths, accomplishments, resources, and goals and to develop plans for addressing immediate needs upon release, including enrolling in treatment.

- Conference Call. About one month prior to release, the second session consisted of a telephone conference call with the client's treatment counselor, parole officer, and family members in which the client reviewed his/her discharge plan and received support and encouragement.

- Community Sessions. In the community, the case manager met with clients weekly for three months, followed by three monthly follow-up contacts for any client needing additional help.

TCM and Standard Referral groups both started from a common 'platform' at each prison treatment site. In both conditions, clients participated in substance abuse treatment in prison and received a referral to publicly funded community treatment. In addition, both groups were shown a professionally produced videotape that was intended to increase the motivation of prisoners to enter treatment following release ("Success Stories II: Part I, Release Day: Where do you go?," FMS Productions, Inc.). 
Case manager characteristics and training

Across the four participating sites, 12 case managers delivered the intervention. Of these, seven were women and five were men. The average age was 39.6 years (range 31-55). By ethnicity, six were African American and six were white. Seven had a bachelor's degree and five a master's degree, with three also having counseling certification. Seven had experience as a case manager before becoming involved in the TCM study (mean=2.5 years; range $=1-6$ years). All sites except one experienced turnover in the case manager position over the course of the study.

The Lead Center for the study developed a detailed manual to provide training for case managers and to promote consistency in implementation across case managers and sites. Topics covered in the manual included the rationale for TCM; description of the two study conditions; detailed procedures for implementing TCM; study monitoring procedures; descriptions of forms and data collection procedures; session objectives, agendas, and sample scripts; job description for the case manager; and general information on research procedures and human subjects issues.

To facilitate fidelity to TCM, case managers received intensive training and regular supervision during the study. A comprehensive three-and-a-half-day training was conducted prior to study initiation. The training included both clinical and research topics, including review of the TCM study and the use of study instruments; an introduction to strengths case management; details on conducting the intervention; and clinical issues in client-case manager interaction. To train case managers hired later in the study, the sites used printed materials and a videotape of the initial training. Once the study was underway, conference calls that focused on case manager questions and issues were conducted bi-weekly initially and then monthly. Site-specific supervision of case managers was conducted regularly. Half-way through the study, a three-hour refresher training for the case managers was conducted via a conference call. The caseload for each case manager was expected to be 15 , although it varied over time, being smaller when the study was beginning and winding down, but reaching 20 at other times depending on case flow.

\section{Methods}

\section{Hypotheses}

The primary goal of providing case management services was to increase the likelihood that parolees would enroll in substance abuse treatment and access other needed services in the community. Through increased participation in substance abuse treatment and receipt of other services, it was expected that clients in the TCM group would have improved drug use, crime, and other outcomes relative to clients in the SR group. Based on these goals, the following hypotheses were tested:

\section{Services Outcomes}

H1 Parolees in the TCM group will be more likely than those in the SR group to enter community substance abuse treatment. 
H2 Of parolees who enter community substance abuse treatment, those in the TCM group will be more likely than those in the SR group to have more days in treatment.

H3 Parolees in the TCM group will be more likely than those in the SR group to receive social services for needs other than substance abuse problems.

H4 Of parolees who receive social services, those in the TCM group will be more likely than those in the SR group to receive a larger amount of a given service.

\section{Behavioral Outcomes}

H5 Parolees in the TCM group will be less likely than those in the SR group to use alcohol and illicit drugs.

H6 Parolees in the TCM group will be less likely than those in the SR group to be re-arrested.

H7 Parolees in the TCM group will be less likely than those in the SR group to report high-risk behaviors related to HIV transmission.

\section{Recruitment and randomization}

Subjects for the study were recruited from secure correctional facilities that offered substance abuse treatment. The number of facilities for recruitment across four research centers (each representing a separate state) varied from 3 to 15. Study recruitment began in November 2004 and ended in March 2007. The CONSORT chart (Fig. 1) depicts the flow of participants through the various stages of the TCM study, from eligibility screening to follow-up. To be eligible for the study, inmates (men and women) within the participating institutions needed to meet the following inclusion and exclusion criteria:

Inclusion criteria Age 18 years or older; enrolled in a drug treatment program within a correctional institution (prison, work release, community correctional facility); had a referral to a community-based substance abuse treatment program; about three months from release; and scheduled for release to a metropolitan area where TCM case managers were located. In each of the participating states, participation in community treatment was mandated, although the degree to which the mandate was enforced varied from state to state. In accordance with human subjects requirements, attendance at TCM case management sessions was voluntary. (In one of the sites, a small number of study participants were under probation supervision upon release from the institution. For convenience, the criminal justice status of the sample is referred to as 'parolee' throughout the paper.)

Exclusion criteria Referral to case management services in the community (e.g., for offenders with co-occurring disorders); registered sex offender; parole requirements that would prevent participation in the study (e.g., Immigration and Customs Enforcement hold for deportation); or inability to provide informed consent. Although inmates were not eligible if it were known at recruitment that they would be receiving (non-TCM) case management upon parole, inmates who were assigned 


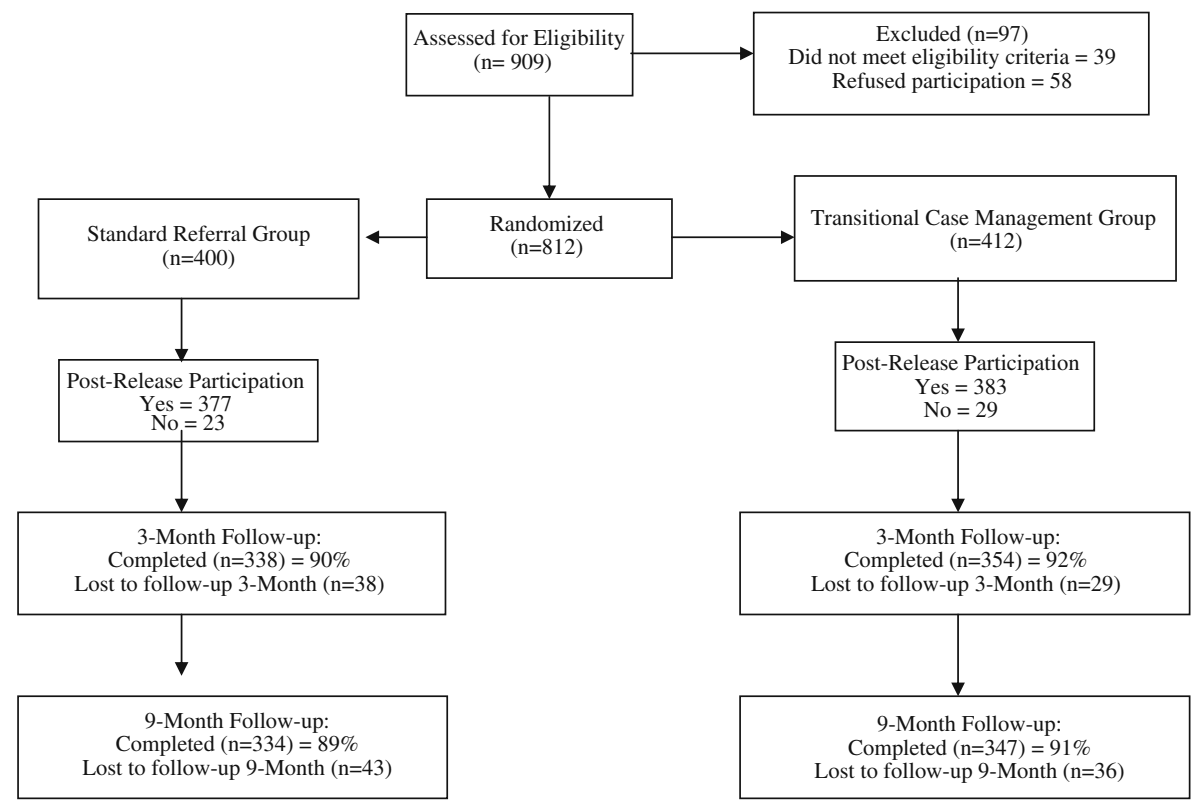

Fig. 1 Flowchart of participants in TCM study. Due to a randomization error, one participant randomized to the SR group was placed in the TCM group. No post-release participation = participants who were not released from prison in time to participate in parole or TCM services and those who paroled to a county or state where TCM services were not available. One participant assigned to the Standard Referral Group was subsequently found to be a sex offender, an ineligibility criterion. This person is included in the no postrelease participation category

to the Standard Referral group could still have received case management services once they were in the community.

Trained research staff from the participating Research Centers contacted the inmates individually, met with them in a private room, confirmed eligibility, described the study and informed consent procedures, and answered any questions. If the inmate agreed to participate, he or she signed the informed consent form. Of those to whom the interviewer described the study and informed consent, $6.7 \%$ refused to participate in the study, prior to being randomized (see Fig. 1). The baseline interview occurred at the time of consent or within the following week.

After completing the baseline interview, clients were assigned to the TCM group or to the Standard Referral group using urn randomization (Hedden et al. 2006; Stout et al. 1994). Urn randomization is a dynamic process that adjusts the probability that a given subject with selected covariates related to outcome is assigned to one of the study conditions based on the characteristics and condition of assignment of previous subjects. As a result, the assignment process is systematically weighted toward maintaining balance on the covariates while retaining randomization as the primary assignment process. The following covariates collected at baseline were used in urn randomization: gender, race/ethnicity, risk for recidivism, substance abuse severity, and type of institutional treatment.

Study procedures and informed consent forms received approval from the CJDATS Steering Committee and from the Institutional Review Board at each of the 
participating Research Centers. A CJDATS Data and Safety Monitoring Board conducted a quarterly review of study implementation and adverse events.

\section{Study sample}

The four Research Centers recruited 812 clients, with each center contributing about 200 clients. Although women comprise about $10-12 \%$ of the correctional population, to have a sufficient number of women for subgroup analyses, the recruitment strategy was designed such that about $25 \%$ of the total sample would be women.

Table 2 presents data comparing participants randomized to the TCM group or to the SR group on selected characteristics at baseline. The two groups did not differ significantly on any of the variables, except for ethnicity, where the TCM group had a larger percentage of whites than did the SR group (50 vs. 44\%). The demographic, drug use, and criminal justice characteristics of the participants were similar to those found in other research samples of substance-abusing prisoners, except that, by design, women were over-represented relative to their percentage in the prison population.

Initially, 412 study participants were assigned to the TCM group and 400 to the Standard Referral group. One subject was subsequently found to be ineligible (sex offender) and was dropped from the study. One client assigned to the Standard Referral group mistakenly ended up in the TCM group. At the time of recruitment, all study participants were scheduled to parole within about three months to a county where they could participate in the TCM study, but subsequently 52 of them (29 in the TCM group and 23 in the SR group) either had their release date changed such that they were not released in time to receive services in the community before the study ended or were scheduled to be paroled to a county or state where TCM services were not provided. These clients were not tracked for follow-up interviews and thus not included in calculations of follow-up rates. These 52 individuals who were excluded prior to release did not differ significantly from the rest of the sample on the variables in Table 2 (based on Fisher's exact test, Pearson chi-square, or $t$ test, as appropriate).

\section{Assessment procedures}

\section{Data collection}

Individual interviews were conducted at three time points by trained research staff: at baseline (shortly after recruitment and approximately three months prior to release from the institution) and at three and nine months following release to parole. Those clients who were not interviewed at three months but were located and interviewed at nine months (36 in the SR group, 26 in the TCM group) were administered an abbreviated version of the three-month interview that omitted questions that referred to 'current' or to "past 30 days," since such questions were captured on the nine-month interview. The remaining questions on the abbreviated three-month interview asked the client to provide responses for the three-month period following release to parole. 
Table 2 Characteristics of TCM group and Standard Referral group at baseline

\begin{tabular}{|c|c|c|}
\hline \multirow[t]{2}{*}{ Variable } & \multicolumn{2}{|c|}{ Baseline $\%$ or mean (SD) } \\
\hline & $\operatorname{SR}(n=400)$ & TCM $(n=412)$ \\
\hline Age & $33.5(9.3)$ & $33.6(8.9)$ \\
\hline \multicolumn{3}{|l|}{ Gender } \\
\hline Male & 78.3 & 73.7 \\
\hline Female & 21.7 & 26.3 \\
\hline \multicolumn{3}{|l|}{ Race/ethnicity } \\
\hline Hispanic/Latino & 16.0 & 14.8 \\
\hline African American/Black & 34.7 & 29.0 \\
\hline White* & 44.4 & 50.1 \\
\hline Asian & 0.5 & 0.7 \\
\hline Native American & 2.2 & 3.2 \\
\hline Other & 2.2 & 2.2 \\
\hline \multicolumn{3}{|l|}{ Marital status } \\
\hline Never married & 53.4 & 57.6 \\
\hline Married or living as married & 18.5 & 16.3 \\
\hline Separated/divorced/widowed & 28.2 & 26.1 \\
\hline Living w/ spouse or partner & 60.0 & 60.1 \\
\hline Homeless & 6.0 & 8.5 \\
\hline Highest grade completed & $10.9(1.9)$ & $10.8(2.0)$ \\
\hline Worked in past 6 months & 50.4 & 47.0 \\
\hline Drug dependence & 82.5 & 83.7 \\
\hline \multicolumn{3}{|l|}{ Primary drug } \\
\hline Opiates & 10.7 & 10.4 \\
\hline Cocaine or crack & 26.1 & 23.0 \\
\hline Methamphetamine & 24.6 & 26.0 \\
\hline Marijuana & 13.2 & 15.1 \\
\hline Alcohol & 22.8 & 21.3 \\
\hline Other & 0.5 & 1.0 \\
\hline Desire for help (range 10-50) & $41.1(6.7)$ & $40.9(7.8)$ \\
\hline Times in jail (lifetime) & $17.3(20.2)$ & $17.0(21.2)$ \\
\hline Months incarcerated (lifetime) & $66.6(51.0)$ & $68.0(55.0)$ \\
\hline Number of arrests (lifetime) & $21.1(22.2)$ & $20.8(22.6)$ \\
\hline Drug-related arrests (lifetime) & $13.5(14.1)$ & $13.0(13.9)$ \\
\hline
\end{tabular}

SR Standard Referral, TCM Transitional Case Management $* p<.05$

Participants received payments for completing research interviews of the same value across all sites. For the baseline interview, clients either had $\$ 10$ deposited to their prison account or received the incentive following release from prison, depending on institutional requirements. They received $\$ 25$ for completing each of 
the three- and the nine-month assessments. Those who completed both the threemonth and the nine-month assessment received an additional $\$ 10$. In addition, those who voluntarily provided a urine sample received $\$ 5$ at each follow-up assessment. The form of payment for the follow-up interviews varied across sites; some provided cash or check, others provided gift cards.

\section{Instruments}

Although a comprehensive set of instruments was administered at baseline and follow-up, of relevance to the analyses reported here are the following instruments (all study instruments are available at http://www.uclapcrc.org/).

The CJ-DATS Core Intake asks for information on sociodemographic background, family and peer relations, health and psychological status, criminal history and criminal justice system involvement, drug use history, and HIV/AIDS risk behaviors. A modified version of this form was used in the follow-up interviews.

The TCU Drug Screen provides an indication of abuse or dependence based on DSM IV criteria, and has been shown to have high levels of positive predictive value, sensitivity, and overall accuracy (Peters et al. 2000).

The Client Evaluation of Self at Intake (CESI) (Simpson and Joe 1993) includes four scales for treatment motivation (problem recognition, desire for help, treatment readiness, external pressure).

The Services Needed and Received form, administered at the two follow-up interviews, collects self-report data on services that clients needed and services that they received since release (three-month follow-up) or since the previous interview (nine-month follow-up). For each of nine types of services, clients were asked how many units (e.g., nights, sessions, visits) they received. Apart from asking clients which services they accessed and how often, we did not collect information on the nature and the quality of the services in the study locations that clients might have been able to receive.

A voluntary urine sample was collected from clients at each follow-up interview.

\section{Follow-up rates}

The three-month follow-up rate across all centers was $91 \%$, with the rate at specific centers ranging from $85 \%$ to $94 \%$. The overall follow-up rate at nine months was $90 \%$, with the center rate ranging from $87 \%$ to $92 \%$. The refusal rates at three months were $2.6 \%$ for the TCM group and $4.7 \%$ for the SR group; at nine months, the rates were $6.3 \%$ and $4.7 \%$, respectively, based on the number due for an interview and contacted at each interview point. The follow-up rate was somewhat higher for clients in the TCM group than for those in the SR group (see CONSORT chart, Fig. 1). This is also reflected in differences in time to follow-up. At three months, the average number of days to the interview was 128.2 $( \pm 77.5)$ for the TCM group and $149.3( \pm 91.7)$ for the SR group $(p=.001)$. These averages include three-month interviews that were conducted at nine months. For the nine-month follow-up, the difference in time to interview was smaller and not 
significant: an average of $304.5( \pm 69.4)$ days for the TCM group and $310.8( \pm 75.4)$ days for the SR group.

Given the high follow-up rate, it is not surprising that the balance between the TCM and the SR groups on client characteristics at baseline was maintained at follow-up. When those who were followed up were compared on baseline variables with those who were not, there were no significant differences at three months. At nine months, there were significant differences on two variables. Those who were interviewed at nine months were more likely than those who were not interviewed to report a history of prostitution or pimping (15.9 vs. $5.4 \%$ ) and to report a lifetime history of crack cocaine use (59.7 vs. $45.7 \%$ ). Comparison of those interviewed for follow-up in the TCM group and the SR group on the same baseline variables indicated only two differences. At the three-month follow-up, the TCM group was significantly more likely than the SR group to have reported lifetime GHB use (4.6 vs. $1.8 \%$ ), and at nine months, the TCM group was significantly more likely to have reported lifetime suicidal ideation (14.7 vs. $9.1 \%)$.

Statistical analyses

\section{Measures}

The independent variable for all analyses is treatment condition, TCM versus SR.

The outcome variables (based on self report) are those specified in the hypotheses listed above, namely:

Substance abuse treatment services (1) Receipt of substance abuse treatment in the community within the interview period, and (2) the number of nights (for residential treatment) or sessions (for outpatient treatment) receiving substance abuse treatment within the interview period. (For the three-month assessment, the period specified for response was the three months following release to parole; for the nine-month assessment, the period was the six months encompassing months four through nine.)

Other social services (1) Receipt of services (other than substance abuse treatment) within the interview period, and (2) for each of nine types of service, the number of service units (variously defined) received within the interview period.

Drug use (1) Any illicit drugs use during the 30 days prior to the interview, and (2) the maximum number of days using any illicit drug during the same time period.

Alcohol use (1) Any alcohol use during the 30 days prior to the interview, and (2) the number of days drinking alcohol during the same time period.

Arrest (1) Any arrest within the interview period, and (2) the number of times arrested within the interview period. 
$H I V$ risk behavior (1) Any sex without a condom during the 30 days prior to the interview, and (2) the number of times having sex without a condom during the same time period.

\section{Analytic approach}

Comparisons of substance abuse and other services received were assessed using chi-square tests for categorical variables and $t$ tests for continuous variables. To determine whether there were differential effects between the TCM and the SR groups over the three observation points on drug use, arrest, and HIV risk outcomes, we examined several two-way interactions for each outcome variable (time by group, with TCM group coded as 1, and SR group coded as 0) using a mixed effects model (Bryk and Raudenbush 1992; Hedeker et al. 1989) with random trends (treating intercept and time as random variables). When applying mixed effects models to longitudinal data, individuals are level two data, and observations within each individual are level one data. A significant two-way interaction would imply that effects over time are different depending on whether participants were assigned to the TCM or SR group. SAS NLMIXED procedures (SAS Institute 2008) with the Bernoulli distribution link were used for binary outcome variables (e.g., any drug use, any arrest). For continuous variables with extremely non-normal distributions including many zeros (e.g., number of arrests), we applied mixed-effects, mixed-distribution models using SAS Macro MIXCORR, developed by Tooze et al. (2002). Mixed-effects, mixed-distribution models incorporate random coefficients in the two-part model, which applies one equation to predict the probability of whether an event happened (e.g., no arrest vs. any arrest) and a second equation to estimate the extent of the non-zero values (i.e., the number of events). This approach also links the two parts through correlated random coefficients (Lambert 1992). To control for days at risk, we included a covariate measuring the total number of days not spent in an institutional setting (inpatient hospital or jail), and treated this as a level-one time-varying covariate. Since the TCM and the SR groups differed significantly on race at baseline, we also include white $(=1)$ versus other $(=0)$ as a level two covariate.

\section{Results}

\section{Session attendance}

Because the effectiveness of the intervention may be influenced by the 'dose' of case management received, the degree to which clients in the TCM group participated in scheduled sessions is important. Since the foundation of case management activities in the TCM study involved clients completing the Strengths Assessment to document accomplishments, strengths, and assets, it was essential that this session take place. Nearly $97 \%$ of the clients did complete the Strengths Assessment with the case manager. In the large majority of cases, the Strengths Assessment occurred in prison shortly after recruitment, but in a few cases, where the client was released 
from prison earlier than expected, the case manager completed the Strengths Assessment with the client at the initial community session.

The Conference Call was intended to provide an opportunity for the client and the other people involved in the client's transition from prison to community to discuss the client's discharge plan, to provide encouragement to attend community treatment, and to identify sources of community support. Across sites, $72 \%$ of the clients participated in the conference call. Scheduling the calls often proved difficult. In some cases, inmates were paroled before the call could take place. In other cases, institutional staff or parole officers declined to participate. Equipment malfunction prevented some calls from occurring.

The intervention protocol called for 12 weekly sessions between the case manager and the client (in person or by telephone), although additional sessions could occur if needed. We set four sessions as the minimum desired level of participation in the community phase. Overall, over two-thirds (69\%) of the clients attended at least four community sessions; $18 \%$ attended between one and three sessions; and 13\% attended no sessions. The average number of community sessions attended was 5.7 $(\mathrm{SD}=3)$. (A more detailed examination of client adherence in the TCM study is provided in Prendergast et al. 2009.)

Fidelity to strengths model

As an indicator of the degree to which case managers adhered to the strengths model of case management (as opposed to generic case management), we calculated client responses to the Strengths Model Assessment form. The form consists of 11 statements (adapted from Marty et al. 2001) about practices that feature prominently in the strengths case management model (e.g., "My case manager and I have used my strengths and achievements as we develop objectives and activities," "The longterm goals that my case manager and I have set up reflect what I want"). At the three-month follow-up interview, clients in the TCM condition completed the Strengths Model Assessment. It was also administered to those clients in the SR condition $(n=55)$ who reported that they had received other types of case management services in the three months following release. The mean total score (based on summing the item responses), where the maximum possible value is 55, was 47.0 for the TCM group and 42.8 for the SR group $(p=.01)$, suggesting that the experience of TCM clients was more reflective of strengths model practices than was that of Standard Referral clients who received case management in other contexts. In addition, on a short version of the Working Alliance Inventory (Neale and Rosenheck 1995) administered to the TCM clients and to those SR clients who reported receiving case management services, the average score (where the maximum was 5) for TCM clients was 4.36 and for the SR clients, $3.99(p<.001)$, suggesting a relatively good relationship between the strengths-based case managers and their clients.

\section{Validity of self-report}

Clients interviewed for the three- and nine-month assessments were asked to provide a voluntary urine sample, which was analyzed onsite with the AutoSplit ${ }^{\circledR}$ KO $^{\text {TM }}$ SixPanel Test Cup, which tested for amphetamine, methamphetamine, cocaine, THC, 
opiates, and benzodiazepines. A urine sample was not requested of clients who were interviewed in jail or prison or by telephone. Of the 705 clients who were interviewed at three months, 638 had a form on file reporting the results of the urine collection and test procedures. Of these, $67.6 \%$ provided a urine sample. Reasons for failure to obtain a sample were: interview conducted in jail or prison, 92; client refused, 64; client unable to provide sample, 32; and 'other' reasons (includes telephone interviews), 17. At nine months, 681 clients were interviewed, and records on urine collection were available for $675 ; 58.1 \%$ provided a urine sample. Reasons for not obtaining a sample were: interview conducted in jail or prison, 194; client refused, 45; client unable to provide sample, 22; and 'other' reasons, 22.

Table 3 shows the results comparing self-report drug use (last 30 days prior to the interview) and the results of the drug test. In conducting this analysis, a self-report of drug use and a negative urine result were treated as agreement since urine testing cannot detect drug use over the full 30-day self-report period. The sample is based on all of those who had a urine drug test form at each assessment point. As seen in Table 3, the kappas comparing self-report with urine test results of drug use are not significantly different between the TCM and the SR groups at the three-month or the nine-month follow-up. The results at nine months do not change substantially when three-month follow-ups conducted at nine months are omitted (results not shown). The kappas are in the 'substantial agreement' range (0.61-0.80) (Viera and Garrett 2005). Because of this substantial agreement and because data from self-report responses are more complete than from urine test results, the self-report data are used in the analysis of drug use outcomes.

Outcomes at three and nine months

\section{Services}

The primary goal of providing case management services in the TCM study was to increase the likelihood that parolees would enroll in substance abuse treatment

Table 3 Comparison of self-report of drug use (past 30 days) and urine test results among clients who provided a urine specimen

\begin{tabular}{lllll}
\hline & $\mathrm{K}$ & $95 \% \mathrm{CI}$ & $\%$ agreement & $\mathrm{H}_{0}: \mathrm{\kappa}_{\mathrm{SR}=\mathrm{K}_{\mathrm{TCM}} \text { (p-value) }}$ \\
\hline 3-month FU (n) & & & & \\
SR (204) & 0.79 & $0.70,0.88$ & 91.67 & \\
TCM (227) & 0.80 & $0.71,0.89$ & 92.07 & 0.864 \\
Overall (431) & 0.79 & $0.73,0.86$ & 91.88 & \\
9-month FU (n) & & & & \\
SR (184) & 0.90 & $0.82,0.97$ & 95.53 & 0.063 \\
TCM (208) & 0.79 & $0.71,0.88$ & 90.39 & \\
Overall (392) & 0.85 & $0.80,0.91$ & 92.86 & \\
\hline
\end{tabular}

Positive self-report and negative urine test were treated as agreement

SR Standard Referral, TCM Transitional Case Management, FU follow-up 
services and access other needed services. Through increased participation in substance abuse and other services, it was expected that clients in the TCM group would have improved drug use, crime, and other outcomes relative to clients in the SR group. With few exceptions, however, outcomes for clients in the TCM group were not statistically different from those in the SR group. Table 4 shows results comparing the services received by the TCM and the SR groups over the three months following release to parole and over the six months between the three-month and the nine-month interview.

With regard to participation in services, most outcomes for clients in the TCM group were not statistically different from those in the SR group. Receipt of substance abuse treatment in the initial three-month period was reported by $62.1 \%$ of clients in the TCM group and by $65.1 \%$ of clients in the SR group, but the difference was not significant. The predominant treatment service was in an outpatient program, with group counseling. Few clients reported receiving residential treatment or individual counseling. With regard to the amount of treatment received, those in the TCM group reported significantly more nights in residential treatment than did those in the SR group (a mean of 83.1 vs. 62.0). The groups did not differ in the

Table 4 Services received during three months following release to parole and during the six months between the three-month and nine-nonth interview by condition

\begin{tabular}{|c|c|c|c|c|c|c|c|c|}
\hline & \multicolumn{4}{|c|}{$\begin{array}{l}\text { Three months following release to } \\
\text { parole }\end{array}$} & \multicolumn{4}{|c|}{$\begin{array}{l}\text { Six months between the three- } \\
\text { month and nine-month interviews }\end{array}$} \\
\hline & \multicolumn{2}{|l|}{ SR } & \multicolumn{2}{|l|}{$\mathrm{TCM}$} & \multicolumn{2}{|l|}{ SR } & \multicolumn{2}{|l|}{$\mathrm{TCM}$} \\
\hline & $\begin{array}{l}\text { Mean (SD) } \\
\text { or } \%\end{array}$ & $n$ & $\begin{array}{l}\text { Mean (SD) } \\
\text { or } \%\end{array}$ & $n$ & $\begin{array}{l}\text { Mean (SD ) } \\
\text { or } \%\end{array}$ & $n$ & $\begin{array}{l}\text { Mean (SD) } \\
\text { or } \%\end{array}$ & $n$ \\
\hline \multicolumn{9}{|l|}{ Substance abuse treatment services } \\
\hline Any substance abuse treatment received & $65.1 \%$ & 345 & $62.1 \%$ & 360 & $42.8 \%$ & 334 & $47.3 \%$ & 346 \\
\hline Nights of residential treatment & $62.0(38.1)^{*}$ & 31 & $83.1(20.8)$ & 34 & $70.8(49.6)$ & 39 & $93.6(63.0)$ & 41 \\
\hline Sessions of outpatient individual & $11.3(10.2)$ & 23 & $7.9(6.6)$ & 29 & $10.2(9.8)$ & 10 & $10.0(9.1)$ & 18 \\
\hline Sessions of outpatient group & $15.6(10.2)$ & 169 & $15.7(11.8)$ & 155 & $19.7(14.6)$ & 94 & $19.8(15.2)$ & 107 \\
\hline \multicolumn{9}{|l|}{ Other services } \\
\hline Any other service received & $80.7 \%$ & 336 & $80.6 \%$ & 350 & $64.7 \%$ & 334 & $68.8 \%$ & 346 \\
\hline Housing: nights of service & $80.8(11.1)$ & 4 & $60.7(36.3)$ & 9 & $82.2(17.4)$ & 5 & $75.0(21.2)$ & 2 \\
\hline HIV/AIDS: visits & $1.8(1.2)$ & 9 & $3.9(5.0)$ & 8 & $1.3(0.5)$ & 9 & $1.3(0.8)$ & 14 \\
\hline Physical health: nights of service & $1.5(1.0)$ & 4 & $6.7(4.9)$ & 3 & $3.00(2.6)$ & 5 & $3.67(3.8)$ & 3 \\
\hline Physical health: visits & $3.2(4.5)$ & 74 & $2.2(2.2)$ & 85 & $3.24(3.9)$ & 79 & $3.29(3.8)$ & 84 \\
\hline Mental health: nights of service & $8.0(8.5)$ & 2 & $9.3(4.6)$ & 4 & $14.0(0.0)$ & 1 & $14.7(11.7)$ & 3 \\
\hline Mental health: visits & $5.8(8.1)$ & 34 & $5.9(7.9)$ & 32 & $15.6(20.7)^{*}$ & 30 & $7.5(6.4)$ & 29 \\
\hline Relations/family: visits & $7.8(8.9)$ & 19 & $7.1(6.2)$ & 21 & $11.0(7.5)$ & 17 & $14.7(12.5)$ & 17 \\
\hline Employment and education: visits & $10.0(10.9)^{*}$ & 32 & $19.7(27.6)$ & 63 & $12.3(16.9)$ & 28 & $14.0(22.8)$ & 28 \\
\hline Financial: visits & $1.5(0.7)^{*}$ & 101 & $1.8(1.5)$ & 102 & $1.85(1.8)$ & 47 & $2.1(2.0)$ & 66 \\
\hline Legal: visits & $2.1(2.0)$ & 9 & $2.1(1.4)$ & 13 & $1.8(1.0)$ & 10 & $3.0(1.2)$ & 5 \\
\hline
\end{tabular}

Omits those incarcerated 30 or more days during the first three months following release to parole SR Standard Referral, TCM Transitional Case Management

$* p<.05$ 
average number of individual or group counseling sessions attended. No differences in treatment services received were found at nine months.

Clients were also asked about services that they received in areas other than substance abuse treatment, which included housing, HIV/AIDS, medical, mental health, family and social relationships, employment and education, financial, and legal. Over the first three months following release to parole, the percentage who reported any such service was virtually identical in both groups $(80.6 \%$ in the TCM group, $80.7 \%$ in SR group; see Table 4). Over the next six-month period, a somewhat higher percentage of clients in the TCM group reported receiving other services than did clients in the SR group (68.8 vs. $64.7 \%$ ), but the difference was not statistically significant. When asked about the amount of services received in each area, TCM clients reported significantly more units of service at three months than did SR clients in two areas: employment and education (19.7 vs. $10.0)$ and finances (1.8 vs. 1.5). At nine months, the only difference was for the number of mental health services, where the TCM clients reported fewer services than did SR clients (7.5 vs. 15.6). When comparing the TCM and the SR groups within sites, either none of the differences by services for a given site was significant or only one of them was, and then not always in favor of the TCM clients (data not shown).

\section{Behavioral outcomes}

Table 5 presents the results for drug use, crime, and HIV outcomes at baseline, three months, and nine months. The recall period for the three-month interview was the three months following release; for the nine-month interview, the recall period was the six months following the three-month interview. As would be expected due to randomization, the two groups were very similar at baseline on all of the outcomes variables. Both of the groups showed improvement at three and nine months compared with baseline, and nine-month outcomes were higher (less favorable) than were three-month outcomes. Preliminary analysis by site (results not shown) indicated that none of the differences for drug use, crime, or HIV outcomes was significant in any of the sites. Based on the results from the mixed-effects model, none of the between-group differences overall, at either interview point, was statistically significant (results available from the first author).

The analyses presented above were intent-to-treat, in which the opportunity to receive treatment (rather than receipt of treatment) is the causal effect being examined. Such analyses tend to produce conservative results, and while they meet the expectations of researchers concerned about selection bias, they may not be of primary interest or relevance to clinicians, who would like to know the effect of case management for clients who actually participate in at least a minimum number of case management sessions. In subsequent analysis, we compared outcomes (drug use and arrest at three and nine months) for TCM clients who participated in a minimum number of sessions (4) with comparable SR clients using the assumptions and methodology of Complier Average Causal Effect (CACE) analysis (Frangakis and Rubin 2002; Little and Yau 1998).). There were no significant differences between the TCM and the SR groups defined by compliance. (Results of this analysis are available from the first author.) 
Table 5 Drug use, crime, and HIV risk behavior outcome variables by study condition by interview time

\begin{tabular}{lll}
\hline & TCM & SR \\
& Mean (SD) or \% & Mean (SD) or \% \\
\hline
\end{tabular}

Drug use

Any drug use past 30 days

$$
\begin{aligned}
& 3 \text { month } \\
& 9 \text { month }
\end{aligned}
$$

Maximum days using any drug past 30 days

Baseline
3 month
9 month

Any alcohol use past 30 days

Baseline
3 month
9 month

Days drank alcohol past 30 days

$$
\begin{aligned}
& \text { Baseline } \\
& 3 \text { month } \\
& 9 \text { month }
\end{aligned}
$$

Crime

Number of times arrested

$$
\begin{aligned}
& \text { Baseline (past } 30 \text { days) } \\
& 3 \text { month (recall period) } \\
& 9 \text { month (recall period) }
\end{aligned}
$$

$1.02(0.38)$

$0.23(0.52)$

$0.45(0.71)$

$94.3 \%$

$20.9 \%$

$35.7 \%$

$$
\begin{aligned}
& \text { Baseline (past } 30 \text { days) } \\
& 3 \text { month (recall period) } \\
& 9 \text { month (recall period) }
\end{aligned}
$$

HIV Risk Behavior

Any sex without condom past 30 days

$$
\begin{aligned}
& \text { Baseline } \\
& 3 \text { month } \\
& 9 \text { month }
\end{aligned}
$$

$76.0 \%$

$75.6 \%$

$44.3 \%$

$48.2 \%$

$44.2 \%$

$42.5 \%$

Number of times had sex without condom past 30 days

$$
\begin{aligned}
& \text { Baseline } \\
& 3 \text { month } \\
& 9 \text { month }
\end{aligned}
$$

11.33 (23.36)

$9.73(20.30)$

$27.62(39.15)$

$9.11(17.67)$

$12.02(31.40)$

Proportion of days in community (time at risk)

$$
\begin{aligned}
& \text { Baseline (past } 30 \text { days) } \\
& 3 \text { month (recall period } \\
& 9 \text { month (recall period) }
\end{aligned}
$$$$
0.98(0.13)
$$$$
0.97(0.14)
$$$$
0.88(0.27)
$$$$
0.92(0.21)
$$$$
0.86(0.20)
$$

$84.1 \%$

$26.8 \%$

4.97 (2.62)

$0.81(1.86)$

1.36 (4.32)

$70.3 \%$

$26.9 \%$

$12.02(12.49)$

$0.89(1.81)$

$0.86(1.69)$

$1.07(0.64)$

$0.23(0.48)$

$0.45(0.63)$

$92.0 \%$

$20.6 \%$

$38.0 \%$
$0.87(0.21)$ 


\section{Discussion}

This study compared three- and nine-month outcomes of substance-abusing offenders (recruited in prison treatment programs) who were randomly assigned either to strengths-based case management or to referral and supervision as usual. Contrary to expectations, the groups did not show statistically significant differences at the two follow-up points with respect to receipt of substance abuse services or to behavioral outcomes related to drug use, arrest, and HIV risk behavior. Those in the TCM group who attended residential treatment report significantly more days in treatment than did those in the SR group, but the number of parolees who participated in residential treatment was small. The TCM group also reported receiving a higher number of employment and educational services and financial services, while those in the SR group received a higher number of mental health services. Overall, the findings suggest that strengths-based case management, as implemented in the TCM protocol, had limited positive impact on the outcomes of substance-abusing parolees compared with those who received standard parole supervision and referral services.

Thus, the results of this study do not support the positive findings from the previous studies of case management in general (Coviello et al. 2006; Rapp et al. 2008; Siegal et al. 1996; Sorensen et al. 2005) and of strengths-based case management in particular (Hall et al. 1999; Siegal et al. 2002; Strathdee et al. 2006; Vaughan-Sarrazin et al. 2000). It should be noted, however, that earlier studies recruited their participants from a general substance-abusing population (although many clients did have a history of criminal justice involvement). The TCM study was the first (to our knowledge) to test the effectiveness of strengths-based case management with an exclusively parolee population recruited in prison.

The case managers in this study made direct referrals to treatment programs and service agencies and assisted clients in overcoming barriers to accessing such services. Although the case managers did provide some support and counseling to help clients deal with personal or interpersonal problems, it was expected that such problems would primarily be addressed when clients sought services in treatment or other agencies. In other words, services were not directly provided by or through the TCM study; it was up to the client, often with the assistance of the case manager, to go to a specific agency if he or she thought that a service would be helpful for a particular need. For this reason, the case managers had an indirect effect on longerterm behavioral outcomes such as drug use, crime, and employment since these outcomes primarily depended on whether clients sought out services and on the nature, quality, and intensity of the services provided by community treatment programs and other service agencies. We did not collect comprehensive data on the service ecology of cities or counties where TCM case management was provided.

Although $69 \%$ of clients in the TCM group attended four or more community sessions, attendance could have been better. Early in the study, it became evident that there was a problem with attendance at case management session. To attempt to correct this, the participating research centers identified specific activities that might increase attendance. Case managers were expected to undertake and document the following activities to re-engage clients who missed two consecutive sessions: write letters; make telephone calls during work hours, evenings, and weekends; attempt to 
locate clients in the community (home, parole office, etc.); and conduct database searches for contact information. The case managers documented each activity for each client and reported aggregate results monthly to the lead center of the TCM study. How effective were these efforts to re-engage clients? When this heightened activity began, $67 \%$ of TCM clients to that point had attended four or more sessions. At the end of the intervention 18 months later, the percentage was $69 \%$, with the percentage for the intervening months never exceeding $70 \%$. It appears that the activities undertaken to improve session attendance had little effect, although they may have prevented a decline in attendance over time. In assessing the success of these efforts, it should be noted that participation in case management was voluntary and that $24 \%$ of the clients were reincarcerated before the end of the 12 -week intervention.

The study was designed to determine whether the TCM intervention improved parolee outcomes compared with standard parole supervision. But various considerations need to be addressed before it can be concluded that this study was a fair test of strengths case management with this population. These considerations fall under three categories: theory, design, and implementation.

With respect to theory, strengths-case management is not a new or an untested intervention. It has a theoretical and empirical history extending back at least 20 years, and case management generally has an even longer history. Principles, techniques, and procedures are documented in research and clinical literature (e.g., Rapp and Wintersteen 1989; Saleebey 2002) and are included in the professional training of case managers. The TCM intervention included the main activities included in all case management models as well as those elements that are unique to the strengths approach to case management. The intervention manual for TCM was adapted from previous manuals on strengths case management developed in NIDAfunded studies.

What was new about the TCM study was its use with a prisoner/parole population that was classified institutionally as low or medium risk. This population presents conditions and circumstances that are not usually faced by case managers who work with a general population of clients with substance use problems and whose criminal justice status is typically probation or drug court. The transition period from prison to the community is extremely stressful for parolees, who have many needs associated with re-entry after several years of incarceration and who may have difficulty addressing them. In addition, since family and other social bonds have often been strained or broken, there may be limited support for recovery and prosocial behaviors. Parole agents, whose primary responsibility is supervision and public safety, may not have the resources or the time to assist with these needs. Under these circumstances, case management principles and practices developed for other populations may need to be adapted to the needs of parolees.

For this population, the main addition of the TCM intervention to the strengths case management approach was scheduling a conference call with the parolee, counselor, parole officer, and family members prior to release. Less than $75 \%$ of these calls took place, and in any case, the calls may have been a weak addition to the standard strengths case management model.

With respect to design, the TCM study had a rigorous design that was well executed. The target sample was 800 , the final sample was 812 . Eligible subjects 
were consented and randomized (by computer at a central location) to one or the other of the study groups. Compliance with assignment was very good. The interview forms included either standardized instruments or instruments used in previous studies with an offender population. Objective measures (i.e., urine tests, official records) were collected. Follow-up rates were high $(91 \%$ at three months, $90 \%$ at nine months). The follow-up points were designed to assess shorter-term (three months) and longer-term (nine months) outcomes. Although researchers often prefer a longer assessment period (12 months or more), it is usually to see whether the early group differences hold up over a longer period (they seldom do, at least for offender populations). The major problem in study design was that 52 inmates who entered the study had their release date extended beyond the point where they could participate in community case management services or were released to counties or states without TCM services. These subjects were not included in follow-up. The number of subjects who did not parole in time was similar in the two study conditions (29 in TCM, 23 in SR) and did not differ from those who were released to parole. Since many of those whose prison stay was extended had violated some rule, the practical effect of not including those not released is that the overall evaluated sample was at somewhat lower risk for recidivism than otherwise.

The eligibility criteria of this study placed few restrictions on who could participate. The main exclusion criteria were whether the person was a sex offender, had an immigration hold, was too cognitively impaired to provide informed consent, or already had a referral to case management following release to parole (which would most likely be indicative of a mental health problem). The fact that only 39 of the 909 prisoners who were formally screened for eligibility were excluded (see Fig. 1) suggests that the individuals who were recruited into the study constituted a heterogeneous sample. It is possible, however, that selecting offenders on the basis of need (using a formal needs assessment procedure) would have resulted in a more appropriate population to receive case management.

Another consideration in assessing influences on study findings is how well the intervention was implemented. Each of the participating research organizations hired (either directly or through subcontract) staff with experience in counseling or case management and in working with clients who were drug users and/or offenders. All case managers received initial and refresher training in the principles and practices of strengths case management and in the procedures of the study. Supervision at each center and monthly conference calls addressed problems and helped promote adherence to the study protocol. Case managers completed forms at each session that indicated whether the expected activities for a given session were completed, partially completed, or not completed. Another form documented non-scheduled contacts with clients or with other persons (e.g., parole agents, service providers, family members). The participating centers received regular quality assurance reports from the lead center that listed missing forms or missing responses that the case manager were expected to correct. These forms continually reminded the case managers of the elements of the study protocol that they needed to comply with. The turnover in case managers at three of the four sites may have had an effect on outcomes, despite efforts to provide training in the model to the new case managers. Although all studies experience problems in implementation, the TCM study was carried out with a high degree of fidelity to its original protocol. 
Client attendance at case management sessions is likely to have affected outcomes, although it is unclear what an adequate 'dose' of case management would be. Nearly $70 \%$ of TCM clients attended four or more case management sessions. This is comparable to the actual number of sessions that clients have attended in other intervention studies, including those of case management (see Prendergast et al. 2009 for an analysis of compliance in the TCM study). As noted above, the TCM case managers took active measures (e.g., telephone calls, letters) to engage clients who missed sessions, but clients ultimately decided how many sessions they wanted to attend - or they were reincarcerated and could not attend any more sessions. With respect to the strengths model itself, compared with SR clients who received other case management services, TCM clients were more likely to agree that they received services in accordance with the principles and practices of strengths case management. One factor that might have affected the outcomes is travel. Although this was not measured, case managers in all participating sites traveled considerable distances to one or more prisons on numerous occasions, and in two of the sites, case managers traveled to other cities to meet with clients away from the central office. Such travel time may have reduced the time that case managers had to spend with clients.

Determining whether adding case management to standard parole services would improve outcomes depends, in part, on whether the TCM group and the SR group received similar parole services (independently of case management). If not, differences (or lack thereof) between the groups may not be directly attributable to case management. Since detailed information on parole services was not collected, a strong test of this factor is not possible. However, a proxy measure of the level of parole services between groups is the response from clients as to who referred them to various services. At the three-month interview, clients in the TCM group reported that $40 \%$ of referrals to services were from their parole officer, compared with 39\% of referrals from parole officers in the SR group, suggesting that parole officers were equally as likely to refer parolees in both groups to services. A related issue is whether clients lacked trust in their case manager over concerns that information provided in sessions would be shared with the parole officer. Case managers made it clear to clients (and to parole officers if the issue arose) that any information that clients provided to the case manager would not be shared with their parole officer without the client's permission.

Although the strengths model of case management used in this study did not improve outcomes overall, modifications to the model might prove beneficial. First, case managers might have contacted inmates earlier and more frequently in prison to establish a stronger relationship and reinforce plans for re-entering the community, although this would add to the cost of the intervention. Second, the physical location of the case manager might be important. In this study, case managers were usually located in stand-alone offices separate from parole, treatment, and other services. Client attendance and services coordination might be improved if case managers were hired by and located at a parole or social services agency. The case managers in all of the sites were located in metropolitan areas; their active assistance in locating and coordinating services with clients might have had greater impact in rural areas, where services are more limited. Third, the 'dose' of the intervention could be increased by lengthening the time that case management is provided (e.g., six months) in order to give the case manager and the client more time to address different needs over a more extended period. But given the high early dropout rate observed in this study (and in most others), 
it is not clear how many clients would take advantage of the additional time. Fourth, greater participation might be encouraged by providing incentives for attendance in accordance with contingency management principles (e.g., Carroll et al. 2006; Helmus et al. 2003). Although incentives need not be costly, they would add somewhat to the cost of a case management intervention. Fifth, case management services could be targeted to a more homogeneous population of parolees with high need (relative to other parolees). Finally, rather than providing case management to parolees who have participated in prison drug treatment programs and who already have a referral to treatment, case management might be more effective with parolees who did not receive prison treatment and who lack connections to community treatment. One or more of these modifications could be tested in a randomized trial to determine whether they have a positive impact on parolee outcomes.

Findings should be considered in light of several limitations. Given the characteristics and circumstances of parolees, the results of the study should not be generalized to other populations with substance abuse problems. Although case managers were provided training, supervision, and feedback on their adherence to the protocol, the study did not use clinical trial methods of fidelity monitoring such as recording and rating case management sessions. The expected and actual dosage of treatment may not have been strong enough to affect outcomes, but it is not clear how much attendance could have been increased given the voluntary nature of the intervention. Measures of services received and behavioral outcomes were based on self-report, except for drug use, where comparison of self-report with urine test results indicated a high degree of concordance.

\section{Conclusion}

Given the importance of increasing parolees' likelihood of successful re-entry, case management would seem to be a potentially useful intervention. For the case management model evaluated in this study, however, on average, the main expected outcomes with respect to linkages with services and longer-term behavioral outcomes were not found for parolees with substance abuse problems, and the few differences that were found were small and of marginal clinical significance. The findings are based on a multisite, randomized design of an established case management approach, both of which were generally well implemented, lending support to the overall validity of the findings. Although case management models have been found to be effective with general substance-abusing clients, particularly with respect to linkages to services, the results from this study should lead to caution in the use of strengths case management with a parolee population. Nonetheless, enhancements to the model examined in this study or the use of other case management models should be examined for their effectiveness with parolee populations.

Acknowledgements This study was funded under Criminal Justice Drug Abuse Treatment Studies (CJ-DATS), a cooperative agreement from the National Institute on Drug Abuse, National Institutes of Health (NIDA/NIH), with support from the Center for Substance Abuse Treatment of the Substance Abuse and Mental Health Services Administration, the Centers for Disease Control and Prevention (CDC), the National Institute on Alcohol Abuse and Alcoholism (all part of the U.S. Department of Health and Human Services); and from the Bureau of Justice Assistance of the U.S. Department of Justice. The authors gratefully acknowledge the collaborative contributions of NIDA, 
the Coordinating Center (George Mason University/University of Maryland at College Park), and the Research Centers participating in CJ-DATS (Brown University, Lifespan Hospitals and Memorial Hospital of Rhode Island; Connecticut Department of Mental Health and Addiction Services; National Development and Research Institutes, Inc.(NDRI) Center for Therapeutic Community Research; the NDRI Center for the Integration of Research and Practice; Texas Christian University, Institute of Behavioral Research; University of Delaware, Center for Drug and Alcohol Studies; University of Kentucky, Center on Drug and Alcohol Research; University of California at Los Angeles, Integrated Substance Abuse Programs; and University of Miami, Center for Treatment Research on Adolescent Drug Abuse). The contents are solely the responsibility of the authors and do not necessarily represent the views of the Department of Health and Human Services, the Department of Justice, NIDA, or other CJ-DATS participants. Thanks are due to Dr. James A. Hall, whose Iowa Case Management Model provided the foundation for the TCM protocol and who provided training to the case managers. Thanks also to Allison Rokke for editorial assistance and to Umme Warda for statistical support. We also thank three anonymous reviewers for comments that improved the paper.

Open Access This article is distributed under the terms of the Creative Commons Attribution Noncommercial License which permits any noncommercial use, distribution, and reproduction in any medium, provided the original author(s) and source are credited.

\section{Appendix}

Reasons that participants were lost to follow-up

\begin{tabular}{lll}
\hline & SR & TCM \\
Three-month follow-up & & \\
Refused & 18 & 10 \\
Deceased & 2 & 1 \\
Deported & 1 & 0 \\
Not located & 11 & 12 \\
Located - not able to interview & 6 & 6 \\
Nine-month follow-up & & 24 \\
Refused & 8 & 1 \\
Deceased & 2 & 0 \\
Deported & 1 & 11 \\
Not located & 18 & 0 \\
Located - not able to interview & 4 & \\
\hline
\end{tabular}

\section{References}

Bryk, A. S., \& Raudenbush, S. W. (1992). Hierarchical linear models: Applications and data analysis methods. Thousand Oaks, CA: Sage.

Carroll, K. M., Easton, C. J., Nich, C., Hunkele, K. A., Neavins, T. M., Sinha, R., et al. (2006). The use of contingency management and motivational/skills-building therapy to treat young adults with marijuana dependence. Journal of Consulting and Clinical Psychology, 74(5), 955-966.

Coviello, D. M., Zanis, D. A., Wesnoski, S. A., \& Alterman, A. I. (2006). The effectiveness of outreach case management in re-enrolling discharged methadone patients. Drug and Alcohol Dependence, 85 (1), 56-65.

de Shazer, S. (1988). Clues: Investigating solutions in brief therapy. New York: W.W. Norton.

Frangakis, C. E., \& Rubin, D. B. (2002). Principal stratification in causal inference. Biometrics, 58, 21-29. 
Gingerich, W. J., \& Eisengart, S. (2000). Solution-focused brief therapy: A review of the outcome research. Family Process, 39(4), 477-498.

Hall, J., Vaughan-Sarrazin, M., Vaughn, T., Block, R., \& Schut, A. (1999). Iowa case management for rural drug abuse: Preliminary results. Journal of Case Management, 1(4), 232-243.

Hall, J., Carswell, C., Walsh, E., Huber, D., \& Jampoler, J. (2002). Iowa case management: Innovative social casework. Social Work, 47(2), 132-141.

Hall, E. A., Zuniga, R., Cartier, J., Anglin, M. D., Danila, B., Ryan, T., et al. (2003). Staying in touch: A fieldwork manual of tracking procedures for locating substance abusers for follow-up studies (2nd ed.). Los Angeles: UCLA Integrated Substance Abuse Programs.

Hall, E., Prendergast, M., Wellisch, J., Patten, M., \& Cao, Y. (2004). Treating drug-abusing women prisoners: An outcomes evaluation of the Forever Free Program. Prison Journal, 84(1), 81-105.

Hammett, T. M., Roberts, C., \& Kennedy, S. (2001). Health-related issues in prisoner reentry. Crime \& Delinquency, 47(3), 390-409.

Hedden, S., Woolson, R. F., \& Malcolm, R. J. (2006). Randomization in substance abuse clinical trials. Substance Abuse Treatment, Prevention, and Policy, 1(6).

Hedeker, D., Gibbons, R. D., Waternaux, C., \& Davis, J. M. (1989). Investigating drug plasma levels and clinical response using random regression models. Psychopharmacology Bulletin, 25(2), 227-231.

Helmus, T. C., Saules, K. K., Schoener, E. P., \& Roll, J. M. (2003). Reinforcement of counseling attendance and alcohol abstinence in a community-based dual-diagnosis treatment program: A feasibility study. Psychology of Addictive Behaviors, 17(3), 249-251.

Hesse, M., Vanderplasschen,W., Rapp, R. C., Broekaert, E., \& Fridell, M. (2007). Case management for persons with substance use disorders. Cochrane Database of Systematic Reviews 2007, Issue 4. Art. No.: CD006265.

Hubbard, R. L., Marsden, M. E., Rachal, J. V., Harwood, H. J., Cavanaugh, E. R., \& Ginzburg, H. M. (1989). Drug abuse treatment: A national study of effectiveness. Chapel Hill, NC: University of North Carolina Press.

Kerson, T. S. (2001). Boundary spanning: An ecological reinterpretation of social work practice in health and mental health systems. New York: Columbia University Press.

Kisthardt, W. E. (2002). The strengths perspective in interpersonal helping: Purpose, principles, and functions. In D. Saleeby (Ed.), The strengths perspective in social work practice (pp. 163-185). Boston: Allyn \& Bacon.

Knight, K., Simpson, D.D., \& Hiller, M.L., (1999). Three-year reincarceration outcomes for in-prison therapeutic community treatment in Texas. The Prison Journal, 79, 337-351.

Lambert, D. (1992). Zero-inflated Poisson regression, with application to defects in manufacturing. Technometrics, 34, 1-14.

Little, R., \& Yau, L. (1998). Statistical techniques for analyzing data from prevention trials: Treatment of no-shows using Rubin's causal model. Psychological Methods, 3(2), 147-159.

Martin, S., \& Inciardi, J. (1993). Case management approaches for the criminal justice client. In J. Inciardi (Ed.), Drug treatment and criminal justice (pp. 84-86). Thousand Oaks, CA: Sage.

Martin, S., Isenberg, H., \& Inciardi, J. (1993). Assertive community treatment: Integrating intensive drug treatment with aggressive case management for hard to reach populations. In J. Inciardi, F. Tims, \& B. Fletcher (Eds.), Innovative approaches in the treatment of drug abuse: Program models and strategies (pp. 87-96). Westport, CT: Greenwood Press.

Martin, S. S., Butzin, C. A., Saum, C. A., \& Inciardi, J. A. (1999). Three-year outcomes of therapeutic community treatment for drug-involved offenders in Delaware: From prison to work release to aftercare. Prison Journal, 79(3), 294-320.

Marty, D., Rapp, C. A., \& Carlson, L. (2001). The experts speak: The critical ingredients of strengths model case management. Psychiatric Rehabilitation Journal, 24(3), 214-221.

Mejta, C., Bokos, P., Mickenberg, J., Maslar, M., \& Senay, E. (1997). Improving substance abuse treatment access and retention using a case management approach. Journal of Drug Issues, 27(2), 329-340.

Miller, S. D., Hubble, M. A., \& Duncan, B. L. (1996). Handbook of solution-focused brief therapy. San Francisco: Jossey-Bass.

Neale, M. S., \& Rosenheck, R. A. (1995). Therapeutic alliance and outcome in a VA intensive case management program. Psychiatric Services, 46(7), 719-723.

Peters, R. H., Greenbaum, P. E., Steinberg, M. L., Carter, C. R., Ortiz, M. M., Fry, B. C., et al. (2000). Effectiveness of screening instruments in detecting substance use disorders among prisoners. Journal of Substance Abuse Treatment, 18(4), 349-358.

Prendergast, M. (2009). Interventions to encourage successful re-entry among drug-abusing parolees. Addiction Science \& Clinical Practice, 5(1), 4-13. 
Prendergast, M., \& Cartier, J. (2008). Improving parolees' participation in drug treatment and other services through strengths case management. Perspectives: Journal of the American Probation and Parole Association, 32(1), 38-46.

Prendergast, M., Greenwell, L., Cartier, L., Sacks, J., Frisman, J., Rodis, E., et al. (2009). Adherence to scheduled sessions in a randomized field trial of case management: The CJ-DATS Transitional Case Management Study. Journal of Experimental Criminology, 5, 273-279.

Rapp, C. A. (1992). The strengths model: Case management with people suffering from severe and persistent mental illness. New York: Oxford University Press.

Rapp, C., \& Wintersteen, R. (1989). The strengths model of case management: Results from twelve demonstrations. Psychosocial Rehabilitation Journal, 13(1), 23-32.

Rapp, R. C., Otto, A. L., Lane, D. T., Redko, C., McGatha, S., \& Carlson, R. G. (2008). Improving linkage with substance abuse treatment using brief case management and motivational interviewing. Drug and Alcohol Dependence, 94(1-3), 172-182.

Ridgely, M., \& Willenbring, M. (1992). Application of case management to drug abuse treatment: Overview of models and research issues. In R. Ashery (Ed.), Progress and issues in case management (NIDA technical review on case management). Rockville: National Institute on Drug Abuse, U.S. Department of Health and Human Services.

Ridgely, M., Morrissey, J., Paulson, R., Goldman, H., \& Calloway, M. (1996). Characteristics and activities of case managers in the RWJ Foundation Program on chronic mental illness. Psychiatric Services, 47(7), 737-743.

Saleebey, D. (1996). The strengths perspective in social work practice: Extensions and cautions. Social Work, 41(3), 296-305.

Saleebey, D. (2002). The strengths perspective in social work practice (3rd ed.). Boston: Pearson Allyn \& Bacon.

SAS Institute. (2008). SAS/STAT Software, Version 8. Cary, NC: SAS Institute.

Siegal, H. A., Rapp, R. C., Kelliher, C. W., Fisher, J., Wagner, J. H., \& Cole, P. (1995). The strengths perspective of case management: A promising inpatient substance abuse treatment enhancement. Journal of Psychoactive Drugs, 27(1), 67-72.

Siegal, H., \& Rapp, R. (Eds.). (1996). Case management and substance abuse treatment: Practice and experience. New York: Springe.

Siegal, H., Fisher, J., Rapp, R., Kelliher, C., Wagner, J., O'Brien, W., et al. (1996). Enhancing substance abuse treatment with case management: Its impact on employment. Journal of Substance Abuse Treatment, 13(2), 93-98.

Siegal, H., Li, L., \& Rapp, R. (2002). Case management as a therapeutic enhancement: Impact on posttreatment criminality. Journal of Addictive Diseases, 21(4), 37-47.

Simpson, D. D., \& Joe, G. W. (1993). Motivation as a predictor of early dropout from drug abuse treatment. Psychotherapy, 30, 357-368.

Simpson, D. D., Joe, G. W., \& Brown, B. S. (1997). Treatment retention and follow-up outcomes in the Drug Abuse Treatment Outcome Study. Psychology of Addictive Behaviors, 11(4), 294-307.

Sorensen, J. L., Masson, C. L., Delucchi, D., Sporer, K., Barnett, P. G., Mitsuishi, F., et al. (2005). Randomized trial of drug abuse treatment-linkage strategies. Journal of Consulting and Clinical Psychology, 73(6), 1026-1035.

Stout, R. L., Wirtz, P. W., Carbonari, J. P., \& Del Boca, F. K. (1994). Ensuring balanced distribution of prognostic factors in treatment outcome research. Journal of Studies on Alcohol. Supplement, 12, 70-75.

Strathdee, S. A., Ricketts, E. P., Huettner, S., Cornelius, L., Bishai, D., Havens, J. R., et al. (2006). Facilitating entry into drug treatment among injection drug users referred from a needle exchange program: Results from a community-based behavioral intervention trial. Drug and Alcohol Dependence, 83(3), 225-232.

Taxman, F. S. (2004). The offender and reentry: Supporting active participation in reintegration. Federal Probation, 68(2), 31-35.

Tooze, J. A., Grunwald, G. K., \& Jones, R. H. (2002). Analysis of repeated measures data with clumping at zero. Statistical Methods in Medical Research, 11(4), 341-355.

Vaughan-Sarrazin, M. S., Hall, J. A., \& Rick, G. S. (2000). Impact of case management on use of health services by rural clients in substance abuse treatment. Journal of Drug Issues, 30(2), 435-463.

Viera, A. J., \& Garrett, J. M. (2005). Understanding interobserver agreement: The kappa statistic. Family Medicine, 37(5), 360-363.

Wexler, H. K., Melnick, G., Lowe, L., \& Peters, J. (1999). Three-year reincarceration outcomes for Amity in-prison therapeutic community and aftercare in California. Prison Journal, 79(3), 312-336. 
Michael Prendergast Ph.D., is Director of Criminal Justice Research Group at the UCLA Integrated Substance Abuse Programs. He has been Principal Investigator of several state and federally funded projects to study drug treatment strategies in the criminal justice system. He has also conducted four metaanalyses on the effectiveness of drug abuse treatment. His research interests include treatment interventions for drug-abusing offenders, systematic reviews of treatment effectiveness, and strategies for implementing evidence-based practices.

Linda Frisman Ph.D., is the Director of Research at the Connecticut Department of Mental Health and Addiction Services, and Research Professor in the University of Connecticut School of Social Work. She has been the Principal Investigator of several federally funded studies testing interventions that address homelessness, co-occurring mental health and substance use disorders, and criminal justice populations with behavioral health disorders. Dr. Frisman holds a Ph.D. in Social Policy from the Heller School of Brandeis University.

JoAnn Y. Sacks Ph.D., is the Executive Director of National Development and Research Institutes, Inc. in New York City. She has been the Principal Investigator on multiple NIH-funded research projects focused on treatment alternatives for individuals with substance use and with co-occurring mental conditions, including those in the criminal justice system. She has authored numerous publications related to this body of research. Dr. Sacks has also been involved in the design, implementation, and evaluation of treatment models for special populations for the past 20 years.

Michele Staton-Tindall Ph.D., is an Assistant Professor in the University of Kentucky College of Social Work and a Faculty Associate at the Center on Drug and Alcohol Research and the Center on the Study of Violence Against Children. Her research interests include women and substance abuse, prison-based substance abuse treatment, service use among incarcerated women, and the impact of substance abuse on children and families.

Lisa Greenwell Ph.D. is a Research Associate at the Portland, Oregon office of RMC Research Corporation. She has considerable experience conducting applied statistical analyses of quantitative data on substance abuse treatment, aftercare, and prevention for a range of population subgroups. She earned a Ph.D. in Sociology from Indiana University and completed a Post-Doctoral Fellowship in Population Studies at RAND.

Hsiu-Ju Lin Ph.D. is currently working as a data analyst for the Research Division at the Connecticut Department of Mental Health and Addiction Services, and a research associate in the University of Connecticut School of Social Work. She has assisted in data analyses of several federally funded studies led by Dr. Linda Frisman. Dr. Lin holds a Ph.D. in Social/Personality Psychology from the University at Albany, State University of New York. Dr. Lin specializes in longitudinal data analysis, multilevel modeling, structural equation modeling, and health psychology studies.

Jerry Cartier M.A., has considerable experience in the drug and alcohol treatment field as a practitioner, administrator, and researcher. Mr. Cartier served as Executive Director of the Thresholds to Recovery treatment program in Santa Barbara, California (1994-1998). After joining UCLA-ISAP in 1998, he was Study Director for a five-year (1997-2002) evaluation of the California Substance Abuse Treatment Facility/State Prison treatment programs. He was a Study Director for the Transitional Case Management Study. He currently serves as an external facilitator for a process improvement intervention as part of the Criminal Justice Drug Abuse Treatment Studies initiative. 